\title{
INDEX OF NAMES
}

The page numbers of review articles are printed in bold type. Short contributions and contributions to discussions are shown in italic type. Literature references not included under the above categories are shown in roman type.

Aarseth, S. J. 252

Abell, G. O. 9, 52, 56, 59, 72, 79-92, 92, 109, 213, 253

Adams, J. H. 349

Ade, P. A. R. 154

Aizu, H. 349

Aldrovandi, R. 335, 336

Alfvén, H. 341, 344, 354

Alvarez, L. W. 349

Aly, J. J. 336-337

Ambartsumian, V. A. 364

Ames, A. 55

Ames, S. 227, 242-243, 245, 248, 251, 360

Anderson, J. L. 178

Apparao, M. V. K. 144, 349

Arnold, J. R. 343

Arnowitt, R. 319

Arons, J. 14

Arp, H. 7, 61-67, 67, 69, 72, 73, 74, 119, 175, 300, $310,313,363,364$

Audouze, J. 197, 213

Baade, W. 48

Bahcall, J. N. 54

Bailey, J. A. 121

Baldwin, J. R. 54

Balko, A. V. 190

Balkowsky, C. 69

Barbierie, C. 62

Bardeen, J. 177, 240, 249, 269, 338, 339

Baschek, B. 199

Battistini, P. 62

Baum, W. A. 29, 48, 53, 71

Beckenstein, J. D. 269

Beckman, J. E. 154

Beery, J. G. 159, 160-161, 213

Behr, C. G. 283

Belinskii, V. A. 261, 263, 273, 275, 320, 326, 330

Bennett, A. S. 94

Berger, B. K. 319, 326

Bertola, F. 29

Bisnovatyi-Kogan, G. S. 220

Bjorkholm, P. 16

Black, D. C. 199

Blair, A. G. 143-155, 155, 157, 162, 195, 213, 359
Bogart, R. S. 86

Bogmolov, E. A. 349

Bohlin, R. C. 22

Böhm-Vitense, E. 56

Bolton, J. G. 97-98, 101, 103

Bondi, H. 291-292, 294

Bonnevier, B. 342

Bonnor, W. B. 39, 216

Borken, R. 351

Bortolot, V. J., Jr. 145

Bottinelli, L. 69

Boughn, S. P. 157, 159-160

Bowyer, C. S. 21-22

Boykin, W. R. 349

Boynton, P. E. 4, 99, 143, 158, 163-166, 166, 195, 213, 305, 359, 361

Bracewell, R. N. 164, 304

Braginski, V. B. 299

Branch, D. 52

Branson, N. J. B. A. 99,125

Brecher, K. 39, 350

Bredekamp, J. 338

Bridle, A. H. 96

Brooke, G. 349

Brown, R. L. 20, 240

Browne, I. W. A. 62-63

Buffington, A. 349

Burbidge, E. M. 41, 61-62, 67

Burbidge, G. R. 41, 61-62, 67, 71, 73, 300, 310, $312,342,350$

Carlitz, R. 195, 205, 208, 248

Caroff, L. J. 147, 152

Carpenter, R. L. 80, 164-166

Carter, B. 269, 285, 288-289, 291-298, 298

Casella, R. S. 268

Caser, S. 335-336

Caswell, J. L. 95

Catlin, L. L. 153

Cesarsky, D. A. 199

Chamaraux, P. 69

Chandrasekhar, S. 92, 230

Chernin, A. D. 217, 223, 227, 232, 237, 241, 251

Chibisov, G. V. 193, 216, 227, 234-237, 241, 246

Christy, R. F. 50 
Chubb, T. A. 15

Cisneros, A. 335

Clark, G. W. 351

Clarke, A. R. 349

Clauser, J. F. 145

Colgate, S. A. 209

Collins, C. B. 5, 276, 281, 283, 291, 294

Conklin, E. K. 4, 52, 159, 160-161, 164, 304, 312

Connolly, L. P. 310

Cooper-Rubin, V. 310

Copson, E. T. 306

Cowan, C. L. 312

Cowsik, R. 27, 144

Crawford, D. E. 112

Crighton, D. G. 241

Crowther, J. H. 95

Dallaporta, N. 247, 248

Damle, S. V. 349

Danese, L. 247

Danziger, I. J. 54

Dashevskii, V. M. 362

Dautcourt, G. 166, 299-315

Davidsen, A. 21-22

Davies, I. M. 96

Davis, M. 255

Davis, M. M. 96, 98, 105, 111-113, 117, 121, 128, 166

Deney, C. L. 349

Deser, S. 319

De Vaucouleurs, G. 47, 49, 51-52, 80-81, 90, $161,213,310,311-312$

Deveny, J. B. 41-42

De Witt, B. S. 298, 319, 322

De Young, D. S. 16, 22, 29, 30

Dicke, R. H. 143, 157, 285, 288-289, 291-293

Dirac, P. A. M. 295, 329

Dismukes, K. 160

Donnelly, R. J. 154

Doroshkevich, A. G. 175, 193, 213-225, 274-275, $279,320,332$

Drake, J. F. 199

Dworak, T. Z. 121

Dyer, C. C. 54

Eardley, L. 320

Eastmond, S. 52

Eddington, A. S. 33, 50, 294-295

Edeskuty, F. 213

Ehlers, J. 283

Einstein, A. 329

Ekers, J. 95

Ekers, R. D. 38

Elliot, H. 349

Ellis, G. F. R. 264, 283

Elvius, A. 341-345, 354

Estabrook, F. B. 283
Evenson, P. 349

Fanaroff, B. L. 103-104, 106

Faulkner, J. 38

Fessenko, D. 253

Field, G. B. 13-28, 29-30, 54, 180, 186-187, 191, $216,222,225,351,362,367$

Flin, P. 97, 121-128

Fomalont, E. B. 96, 105, 111, 121

Ford, W. K. 342

Fowler, W. A. 56, 195, 197, 202, 209, 213

Fram, D. M. 157, 159-160

Frautschi, S. 195, 205, 208, 248

Friedman, H. 15

Frogel, J. A. 54

Fugimoto, Y. 349

Fujimoto, M. 241

Gagarin, Yu. F. 349

Gaisser, T. K. 349

Gamow, G. 227, 241

Garmire, G. P. 351

Gascoigne, S. C. B. 50

Geiss, J. 199

Geller, M. J. 55, 251

Geren, P. 283

Geroch, R. 264, 266

Giacconi, R. 15, 21, 352

Gilbert, F. 15

Ginzburg, V. L. 32,329

Gogolewski, S. 256

Golden, R. L. 349

Goldsmith, D. 22

Golenetski, S. V. 337

Gorenstein, P. 16

Gott, J. R. 22-24, 54

Gouguenheim, L. 69

Gowdy, R. H. 299, 319, 320-326

Gower, J. F. R. 94

Greenhill, J. G. 349

Gregory, St. A. 310

Grigorov, N. L. 349

Grishchuk, L. P. 216, 279, 286, 288, 333

Gulkis, S. 164-166

Gunn, J. E. 22-24, 54, 71

Gursky, H. 15, 21, 27, 352

Guyot, M. 216

Hardy, E. 47, 52-53, 55

Harnden, F. 16

Harries, J. E. 154

Harris, B. 16

Harrison, E. R. 220, 223, 227, 235, 332, 360

Harwit, M. 144-146, 153

Hasegawa, S. 349

Hauser, M. G. 86-89, 92, 158

Hawking, S. W. 5, 158, 263-264, 269, 276, 281. 
283-286, 286, 287-289, 291, 294, 361

Hazard, C. 165

Heckmann, H. H. 349

Heckmann, O. 274

Heidmann, J. 47, 65, 69

Heisenberg, W. 247

Heller, M. 3-11, 281, 286-288

Hellsten, H. 342

Henry, P. S. 159-160

Henry, R. C. 14-15, 22

Herzog, E. 9, 82, 85, 87, 121

Hewish, A. 95

Hiebert, R. D. 153, 213

Higbie, P. 351

Hinder, R. A. 99, 125

Hodge, P. W. 49

Holberg, J. 22

Holden, D. J. 99, 125

Holmberg, E. 47-48, 51, 55, 80

Hooley, A. 29, 95

Houck, J. R. 144-146, 153

Hoyle, F. 61, 195, 202, 209, 353-354

Hubble, E. 47-48, 50, 79

Hughes, R. G. 99, 125

Huizinga, J. S. 154

Humason, M. L. 48, 53, 129, 130-140

Iben, I. 38

Icke, V. 92, 225, 240, 249, 298, 327, 333

Illarionov, A. F. 173, 338

Irvine, W. M. 177

Isaacson, R. A. 299, 302

Ivanova, N. S. 349

Jacobs, K. C. 326

Jaffe, W. 29

Janes, K. 41-42

Jauncey, D. L. 112-113

Jeans, J. H. 216

Jefferts, K. B. 199

Jenkins, E. B. 199

Johnson, H. L. 48

Jones, B. J. T. 227, 241, 243, 245, 248, 360

Jordan, P. 292

Kantowski, R. 54

Kapteyn, J. C. 48

Karachentsev, I. D. 253-254

Kardashev, N. S. 40, 42

Karlson, E. T. 341-345

Karpowicz, M. 9, 82, 85-87

Katgert, P. 96

Katgert-Merkelijn, J. K. 96

Kaufmann, W. J. 303, 306

Kayser, S. E. 50

Kellermann, K. I. 40, 54, 98-99, 103, 105, 111118, 118, 121, 125
Kellogg, E. 15, 21, 352

Kempe, K. 314

Kenderdine, S. 121

Khalatnikov, I. M. 214, 216, 261, 263, 273, 275, $320,326,330$

Kiang, T. 55, 86, 118, 124, 128, 165, 253, 254

King, I. R. 16, 22, 55, 91

Kinter, E. C. 16, 22, 55, 91

Kirshner, R. P. 52

Kirzhnits, D. A. 329

Klein, O. 341-342, 354

Klimek, Z. 3-11, 287

Kobetich, E. J. 27

Kompaneets, A. S. 172

Kondrateva, M. A. 349

Koshiba, H. 349

Kowal, C. T. 9, 52, 82, 85, 87

Kraushaar, W. L. 351

Kristian, J. 71, 300

Kronheimer, E. H. 264

Kuchař, K. 322

Kulikov, V. N. 349

Kundt, W. 248-249

Kurskov, A. A. 227, 229, 235-236, 243

Kurt, V. G. 188, 222

Kwast, T. 8

Lake, R. G. 312

Lampton, M. 22

Lanczos, C. 177

Landau, L. D. 261, 287

Laurent, B. E. 341-345, 354

Lauterborn, D. 50

Layser, D. 177, 183, 249-250

Lea, S. M. 15

Lemaître, G. 39

Leong, C. 15, 352

Leong, V. 351

Le Poole, R. S. 96

Lequeux, J. 96

Leroy, B. 336

Limber, D. N. 92

Liang, E. 320

Lifshitz, E. M. 175, 181, 214, 216, 228, 248, 261, $263,273,275,282,287,294,320,326,330-331$, 359

Lighthill, M. J. 241

Lindstrom, P. L. 349

Liubushin, A. A. 329

Longair, M. S. 29, 40, 69-71, 93-108, 108-109, $117,118-119,125,166,221-222,256,363$

Loyd, E. G. 153

Lubyanaya, N. D. 349

Lucchin, F. 247, 248

Lukash, V. N. 275, 278, 333

MacColl, M. 143 
McCallum, M. A. H. 283

McClure, R. D. 54

McCrea, W. H. 162, 249

McEwan, N. J. 62-63

McKee, C. F. 14

McNutt, D. P. 145

McVittie, G. C. 40

Machalski, J. 97, 118, 121-128

Malmquist, K. G. 50

Mandel, L. 300

Marar, T. M. K. 349

Marcus, A. H. 129, 135

Marx, G. 357

Maslowski, J. 96-97, 99, 117, 121-128

Matilla, K. 74-75

Matsuda, T. 227, 241

Matteson, J. L. 343

Matzner, R. A. 320

Maurer, R. H. 349

May, R. M. 54

Mayall, N. U. 48, 53, 129-140

Mayer-Harselwander, H. A. 337

Meekins, J. F. 15

Metzger, A. E. 343

Michie, R. W. 186

Miley, G. K. 16

Millea, M. F. 143

Mills, B. Y. 96

Minkowski, R. 53, 135

Mishystin, I. N. 220

Misner, C. W. 176, 271, 274-275, 282, 284, 319327, 327, 333, 362

Mito, I. 349

Moffet, A. T. 199

Moncrief, V. 322-323

Morgan, D. L. 338

Morgan, W. W. 54

Morton, D. C. 199

Muehlner, P. 147-148, 152-153

Münch, G. 92

Murdoch, H. S. 112

Murray, S. 15, 21, 352

Nahm, W. 195, 205, 208, 248

Nanos, M. 159

Nariai, H. 176, 227, 241-243, 245

Nasi, E. 62

Neville, A. C. 94

Neyman, J. 79, 85, 92, 129-140, 253-254, 314

Nicolle, J. P. 336

Nishimura, J. 349

Nolt, I. G. 154

Noonan, T. W. 55-56

North, J. D. 32, 39

Novikov, I. D. 157-158, 162, 165, 216, 223, 240, $245,271,273-281,281-282,320,327,361$

Novokreshenova, S. I. 221
O'Dell, S. L. 73, 300, 312

Ogorodnikov, K. F. 310

Oke, J. B. 53-54, 71-72, 256, 362

Omnes, R. 203, 247, 335-338, 353, 361

Oort, J. H. 55-56, 216, 227

Osborn, W. H. 41-42

Osterbrock, D. E. 54

Ostriker, J. P. 24

Ozernoy, L. M. 73-74, 165, 176, 193, 219, 223, 227-239, 239-240, 241, 243, 245-246, 248, 255, 281,360

Paál, G. 8, 251-253

Page, T. L. 16, 22, 55, 91

Pal, Y. 349

Parijskij, Yu. N. 4, 21, 29, 164-165, 166, 172-173, 213, 222-223, 246, 281, 305

Parker, L. 319, 330

Partridge, R. B. 4, 52, 74, 92, 99, 157-162, 162 , $164-166,195,213,222,240,254-255,256,304$ 305,361

Pasachoff, J. M. 199

Patchett, B. 52

Pauliny-Toth, I. I. K. 98-99, 103, 105, 111-118, $118-119,121,125,363$

Peach, J. V. 40, 53

Pearson, T. J. 95, 99

Pedersen, R. J. 143

Peebles, P. J. E. 55, 74, 80, 86-89, 92, 143, $157-$ $158,177,184,187-188,190-191,193,195-196$, 205, 216-217, 219, 222-223, 227, 235, 240-241, $248,251,255,332$

Penrose, R. 263-271, 271-272, 287-288, 362

Penzias, A. A. 143, 157, 164, 199

Perola, G. C. 16

Persson, S. E. 54

Peters, W. L. 161, 213

Peterson, B. A. 53

Peterson, L. E. 343

Petrosian, V. 14, 31-46, 147, 152, 329

Pikelner, S. B. 59

Pipher, J. L. 153

Pooley, G. G. 95, 112, 121

Porcas, R. 97

Poveda, A. 239

Press, W. H. 250, 300

Preuß, E. 112

Price, P. B. 144

Puget, J. L. 227, 246, 247, 335-338, 339, 343, 353, 361

Purcell, E. M. 13

Pyatunina, T. B. 164, 305

Racine, R. 49

Radostitz, J. V. 154

Rapoport, I. D. 349

Reedy, R. C. 343 
Rees, M. J. 29, 74, 102, 118, 155, 159, 176, 239, 245-246, 256, 283, 299, 359-365

Reeves, H. 197, 199, 213

Refsdal, S. 50

Reinhardt, M. 240

Reiz, A. 55, 80

Rengaragan, T. N. 349

Richter, L. 314

Richter, N. 314

Rindler, W. 33-34

Roach, F. 255

Roberts, M. S. 22, 47, 55, 240

Robertson, J. G. 96

Robson, E. I. 154

Roeder, R. C. 54, 59, 312

Rogers, W. 365

Rogerson, J. B. 199

Roll, P. G. 143, 157

Romano, G. 54

Romanov, V. A. 349

Romero, H. V. 153

Rood, H. J. 16, 22, 55, 91, 309-310

Rothman, C. A. 309

Rowan-Robinson, M. 40, 61, 73, 108

Rubin, J. S. 342

Rubin, V. C. 342

Rudnicki, K. 3-11, 79, 86, 121, 140

Rudnisky, G. M. 221

Ruffini, R. 299

Ruzmaikin, A. A. 220

Ryabenki, V. S. 216

Ryle, M. 94-95, 102, 112, 121-122

Sachs, R. K. $216,283,300,320$

Salpeter, E. E. 31, 34-38, 40-42, 45, 165, 222

Sandage, A. R. 35, 38, 47-56, 70, 129-140, 213 , 223, 225, 251

Sargent, W. L. W. 69-71, 199, 300, 310, 342

Sasao, T. 242

Saslaw, W. C. 86

Sastry, G. N. 16, 22

Sato, H. 227, 241

Sato, T. 164-166

Savenko, I. A. 349

Schatzman, E. 336

Schechter, P. 250

Schein, M. 349

Scheuer, P. A. G. 40, 166, 173, 256

Schmidt, M. 102, 108, 118-119, 221, 253, 255257

Schraml, J. 164

Schramm, D. N. 197, 213

Schucking, E. 274

Schwartz, D. 27

Sciama, D. W. 161, 283, 295, 304

Scott, E. L. 79, 85, 92, 129-140, 140, 254, 314, 362

Seares, F. H. 48
Searle, L. 56, 199

Seligman, C. E. $82,85-86$

Sersic, J. L. 49

Setti, G. 71

Shaffer, D. 105, 111, 121

Shandarin, S. F. 216, 219-222

Shane, C. D. 55, 79, 85, 87-88, 92, 314

Shapiro, S. 200

Shapiro, S. L. 237

Shapley, H. 55

Shepley, L. C. 191

Shimmins, A. J. 98, 101, 103, 109

Shipley, J. P. 213

Shivanandan, K. 144-146

Shklovsky, I. S. 42, 222

Silk, J. 13, 15, 22, 25, 27, 29, 39, 165, 173, 175-193, $193,216,227,237-238,240,242,245,246,249$, $309,338,360$

Smart, N. 22, 55-56

Smith, L. H. 349

Smith, L. L. 255

Smith, M. G. 165

Smoot, G. F. 349

Söderholm, L. 342, 354

Soifer, B. T. 153

Solheim, J. E. 37

Solinger, A. B. 18

Solomon, P. M. 62, 67, 199

Spiegel, E. A. 178

Spinrad, H. 54

Spitzer, L. 199, 343

Stabell, R. 40

Starobinsky, A. A. 319, 327, 330, 333

Stebbins, J. 48

Stecker, F. W. 227, 247, 337, 338, 343

Steigman, G. 173, 203, 213, 248, 333, 338, 344, 347-356

Stein, R. 360

Stewart, J. M. 161, 288, 320

Stokes, R. A. 143

Strittmatter, P. A. $62,67,73,348,351,354$

Sunyaev, R. A. 20, 29, 166, 167-173, 175, 188$189,193,213-225,241,332,338,359-360$

Suszycki, L. 287-288

Swandic, J. R. 22

Swanson, M. D. 79

Swope, H. H. 48

Szekeres, P. 31, 37, 40-41, 45

Szkody, P. 56

Takase, B. 9

Takeda, H. 227, 241

Tammann, G. A. 47-59, 59, 74, 109, 161, 200, 223, 362-363

Tananbaum, H. 15, 21, 352

Tandon, S. N. 349

Tartar, J. 13, 25, 27, 29, 309 
Taylor, B. J. 54

Thaddeus, P. 144-145

Thomas, L. H. 178-179

Thorne, K. S. 158, 300, 321

Thorsos, T. 351

Tifft, W. G. 300, 308, 314

Tinsley, B. M. 40, 54, 222

Tolman, R. C. 269

Tomita, K. 227, 237

Toomre, A. 72

Trautman, A. 3

Trombka, J. I. 337, 343

Tucker, W. H. 18

Turnrose, B. E. 309

Tyson, J. A. 299

Unno, W. 178

Upton, E. K. L. 50

Urbanik, M. 97, 121-128, 162, 225

Valladas, G. 336

van Bueren, H. G. 50

van Rhijn, P. J. 48

van den Bergh, S. 47, 49, 55

van der Kruit, P. C. 16

van der Laan, H. 16, 96

Vedrenne, G. 337

Verma, R. P. 349

Vernon, F. L., Jr. 143

Vickers, D. G. 154

Von Hoerner, S. 92, 108

Wagoner, R. V. 59, 86, 145, 195-210, 213, 245, $256,261,313,360,362$

Wahlig, M. A. 349

Wahlquist, H. D. 283

Wall, J. V. 53, 103

Walsh, D. 97

Wampler, J. 67

Watson, W. D. 199

Weber, J. 299-300

Webster, A. S. 161

Weigert, A. 50

Weinberg, S. 287

Weiss, R. 147-148, 152-153
Weizsäcker, C. F. von. 227, 241, 247

Welch, G. A. 16, 22

Welch, W. 21-22

Westerlund, B. E. 53

Westervelt, P. 288

Weymann, R. 172, 180, 255

Wheeler, J. A. 288, 291, 299, 321, 362

Whitford, A. E. 48

Wild, P. 9, 82, 85, 87, 121

Wilkinson, D. T. 143, 157, 159-161, 255, 304

Will, C. M. 195

Williamson, K. D. 153, 213

Willson, M. A. G. 16

Wilson, R. W. 143, 157, 164, 199

Wingert, D. W. 14

Wirtanen, C. A. $55,87-88,92$

Witzel, A. 112

Wolf, E. 300

Wolfe, A. M. 216, 283, 300

Wolfendale, A. W. 349

Woltjer, L. 71

Woolf, N. J. 199

Yahil, A. 24, 99, 117, 125

Yilmaz, F. 52

Yokio, K. 349

York, D. G. 199

Young, J. W. 54

Yu, J. T. 88-89, 177, 188, 191, 193, 216, 332

Zajac, B. J. 145

Zapolsky, H. 326

Zel'dovich, Ya. B. ix-xi, 20, 29, 33, 54, 59, 67, 74, $108,155,158,165-167,173,175,186,188-189$, 193, 213-225, 225, 236, 241, 245-246, 248, 250 . 255-256, 261, 267, 271-272, 274-275, 281, 284, 299, 319-320, 329-333, 333, 338, 339, 357, 360362,364

Zhuravlev, D. A. 349

Zięba, A. 9, 97, 121-128

Zięba, S. 97, 117, 121-128

Zipoy, D. M. 299, 303, 306

Zonn, W. 129, 137-138

Zuckerman, B. 155

Zwicky, F. 7-9, 79, 82, 84-88, 121, 314 